\title{
The mortality burden attributable to nontrauma fracture for privately insured adults with epilepsy
}

\author{
Daniel G. Whitney $^{1,2}$ (D) | Sarah Bell ${ }^{1} \quad$ Nancy A. McNamara ${ }^{3}$ ｜ Edward A. Hurvitz $^{1}$
}

${ }^{1}$ Department of Physical Medicine and Rehabilitation, University of Michigan, Ann Arbor, Michigan

${ }^{2}$ Institute for Healthcare Policy and Innovation, University of Michigan, Ann Arbor, Michigan

${ }^{3}$ Department of Pediatrics, University of Michigan, Ann Arbor, Michigan

\section{Correspondence}

Daniel G. Whitney, Department of Physical Medicine and Rehabilitation, University of Michigan, 325 E Eisenhower Parkway, Ann Arbor, MI 48108.

Email:dgwhit@umich.edu

\section{Funding information}

American Academy of Cerebral Palsy and Developmental Medicine; University of Michigan Office of Health Equity and Inclusion Diversity Fund

\begin{abstract}
Objective: Individuals with epilepsy have poor bone development and preservation throughout the lifespan and are vulnerable to nontrauma fracture (NTFx) and postNTFx complications. However, no studies have examined the contribution of NTFx to mortality among adults with epilepsy. The objective was to determine whether NTFx is a risk factor for mortality among adults with epilepsy.

Methods: Data from 2011 to 2016 were obtained from Optum Clinformatics Data Mart, a nationwide claims database from a single private payer in the United States. Diagnosis codes were used to identify adults ( $\geq 18$ years old) with epilepsy, NTFx, and covariates (demographics and pre-NTFx cardiovascular disease, respiratory disease, diabetes, chronic kidney disease, cancer). Crude mortality rate per 100 person-years was estimated. Cox regression estimated hazard ratios (HRs) and 95\% confidence intervals (CIs) were determined for mortality, comparing epilepsy and NTFx (EP + NTFx; $\mathrm{n}=11$ 471), epilepsy without NTFx (EP without NTFx; $n=50$ 384), without epilepsy and with NTFx (without EP + NTFx; $n=423$ 041), and without epilepsy and without NTFx (without EP without NTFx; $n=6.8$ million) after adjusting for covariates.
\end{abstract}

Results: The 3-, 6-, and 12-month crude mortality rates were highest among $\mathrm{EP}+\mathrm{NTFx}(12$-month mortality rate $=8.79)$, followed by without EP + NTFx (12month mortality rate $=4.80)$, EP without NTFx $(12$-month mortality rate $=3.06)$, and without EP without NTFx (12-month mortality rate $=0.47$ ). After adjustments, the mortality rate was elevated for EP + NTFx for all time points compared to EP without NTFx (eg, 12-month HR = 1.70, 95\% CI = 1.58-1.85), without EP + NTFx (eg, 12-month HR $=1.41,95 \% \mathrm{CI}=1.32-1.51$ ), and without EP without NTFx (eg, 12-month $\mathrm{HR}=5.23,95 \% \mathrm{CI}=4.88-5.60)$. Stratified analyses showed higher adjusted HRs of 12-month mortality for EP + NTFx for all NTFx sites (ie, vertebral column, hip, extremities), all age categories (young, middle-aged, older), and for both women and men.

Significance: Among adults with epilepsy and compared to adults without epilepsy, NTFx is associated with a higher 12-month mortality rate. Findings suggest that NTFx may be a robust risk factor for mortality among adults with epilepsy. 


\section{K E Y W O R D S}

epilepsy, mortality, nontrauma fracture

\section{\begin{tabular}{l|l}
1 & INTRODUCTION
\end{tabular}}

Individuals with epilepsy typically require long-term treatment with antiseizure medications to prevent seizures. Seizures and antiseizure medications are well established factors for inadequate skeletal development in children, ${ }^{1}$ leading to low bone mineral density and elevated fracture rate among children with epilepsy, in comparison to children without epilepsy. ${ }^{2,3}$ Three to four of five adults with epilepsy have skeletal fragility (eg, low bone mineral density), ${ }^{4,5}$ and fracture risk is considerably elevated among adults with epilepsy compared to the general population (relative risk $=1.7-6.2$ ) ${ }^{6}$

Among the elderly general population, skeletal fragilityespecially nontrauma fracture (NTFx) - is a primary contributor to loss of function, ${ }^{7}$ chronic disease development, ${ }^{8}$ poor quality of life, ${ }^{9}$ and premature mortality. ${ }^{7,10,11}$ Moreover, health status prior to sustaining a fracture is a strong predictor of postfracture health, function, and survival outcomes. ${ }^{7}$ Therefore, adults with epilepsy may have greater risk of postNTFx mortality because of their poor skeletal development and preservation throughout the lifespan and other unhealthful aging processes. Specifically, individuals with epilepsy have greater risk of chronic diseases across biological systems, including cardiometabolic, respiratory, gastrointestinal, liver, and other neurological diseases, as well as a variety of mental health disorders. ${ }^{12-14}$ Furthermore, there is strong evidence to suggest that individuals with epilepsy have greater risk of premature mortality compared to individuals without epilepsy. ${ }^{15}$ However, the contribution of skeletal fragility to health and survival outcomes for adults with epilepsy has not been investigated.

A better understanding of the link between skeletal fragility and mortality risk is needed for this vulnerable population. If skeletal fragility does increase risk for mortality, clinicians caring for patients with epilepsy should be aware of the need for strategies to prevent skeletal fragility and intensively manage NTFx and its related sequelae, with the ultimate goal of maximizing healthful aging throughout the lifespan. Accordingly, the objective of this study was to determine whether NTFx among adults with epilepsy is associated with greater 12-month mortality rate (MR) compared to adults with epilepsy who do not sustain an NTFx and compared to adults without epilepsy who sustain an NTFx. We hypothesized that adults with epilepsy who sustain an NTFx would have a higher post-NTFx 12-month MR compared to both groups, suggesting that (1) NTFx is a risk factor for 12-month mortality among adults with epilepsy and (2) the NTFx-associated 12-month MR is exacerbated for adults with versus without epilepsy.

\section{Key Points}

- Adults with epilepsy have increased risk of NTFx and premature mortality; however, the link between the two is unknown

- This private claims-based study from 2011 to 2016 found that NTFx is a risk factor for mortality among adults with epilepsy

- Among adults with epilepsy, NTFx was associated with a $187 \%, 134 \%$, and $70 \%$ higher adjusted rate of 3-, 6-, and 12-month mortality, respectively

- Among adults who sustained an NTFx, adults with versus without epilepsy had an $11 \%, 29 \%$, and $41 \%$ higher adjusted rate of 3-, 6-, and 12-month mortality, respectively

- NTFx across all sites, and especially lower and upper extremities, was associated with an elevated 12-month mortality rate for adults with versus without epilepsy

\section{2 | MATERIALS AND METHODS}

\section{1 | Data source}

Data from 2011 to 2016 were extracted from the Optum Clinformatics Data Mart Database (OptumInsight), which is an administrative claims database providing information from privately insured beneficiaries who had commercial or Medicare Advantage plans in the USA. ${ }^{16}$ These claimsbased data include all health service utilization (eg, inpatient, emergency department) for each individual throughout enrollment. To maintain patient confidentiality, researchers leveraging the Optum database are allowed either the Date of Death or Socioeconomic Status table. This study used the Date of Death table, and some information regarding socioeconomic status (ie, income, education) was not available. Data were deidentified and the university institutional review board approved this study as nonregulated.

\section{2 | Sample selection}

All medical conditions (eg, epilepsy, fracture, comorbidities) were identified using the International Classification of Diseases, Ninth and Tenth Revision (ICD-9 and ICD10), Clinical Modification codes to account for the shift in 
reporting codes on October 1, 2015, as previously described. ${ }^{17}$ Information regarding how diagnoses were made and by whom (eg, primary care physician) was not available in administrative claims data.

Adults $\geq 18$ years of age with epilepsy were identified by at least one inpatient, outpatient, or emergency department claim for "epilepsy and recurrent seizures" (ICD-9, 345 family; ICD-10, G40 family) on at least two different days within 365 days of one another. Information regarding epilepsy etiology, type, or duration of diagnosis was either not available in claims data or not reliably coded (eg, $46 \%$ had "unspecified" epilepsy), thus not allowing for stratification or statistical adjustment for the clinical subtypes of epilepsy. The group of adults without epilepsy included individuals with no claims for "epilepsy and recurrent seizures." Using claims to identify epilepsy has high sensitivity and specificity for clinical populations, ${ }^{18}$ and the $2+$ claim algorithm improves the accuracy of identifying "nonevent" medical conditions over a single claim (eg, fracture is an "event"). ${ }^{19}$ For epilepsy, the $2+$ algorithm has a positive predictive value of $\sim 70 \%$. $^{20}$

We defined NTFx as a fracture without trauma codes (eg, motor vehicle accident) 7 days before to 7 days after the index fracture date, as guided by previous studies. ${ }^{17,21,22}$ Fractures of the vertebral column, hip (including proximal femur), nonproximal femur, tibia/fibula, humerus, ulna/ radius, or unspecified location (ICD-9, 733.1 family, 805813 families, 818-823 families, and 827-829 families; ICD-10, "initial encounter" codes from S12, S22, S32, S42, S52, S72, S82, and M80 families, M84.4, M84.6, and M84.7) were identified by at least one inpatient, outpatient, or emergency department claim from 2012 to 2015. This time frame was selected to allow for at least 1 full year of a "look back" period for those experiencing fracture in 2012 to ascertain baseline comorbidity data and at least 1 full year of follow-up for those experiencing fracture in 2015 for the outcome. Using a single claim to capture fracture has excellent accuracy, with up to $98 \%$ positive predictive value, which is similar to or better than other algorithms (eg, $2+$ claims). ${ }^{23}$

The sample was then categorized based on epilepsy and NTFx status: (1) with epilepsy and NTFx (EP + NTFx), with epilepsy and without NTFx (EP without NTFx), without epilepsy and with NTFx (without EP + NTFx), and without epilepsy and without NTFx (without EP without NTFx). The start date of the follow-up was the index date of NTFx or a randomly assigned date for those who did not sustain an NTFx. For the latter, we used a uniform distribution (visually inspected by S.B.) to randomly assign a date during the individual's enrollment period between January 1, 2012 and December 31, 2015.

We included individuals who had at least 12 full months of continuous enrollment in a health plan prior to their start date of follow-up to sequester baseline comorbidity data, as previously described. ${ }^{19}$

\section{\begin{tabular}{l|l}
2.3 & Outcome measure
\end{tabular}}

All-cause mortality from 2012 to 2016 was determined as the number of days from the start date of follow-up to date of death, and separated as 3-, 6-, and 12-month time points (6 and 12 months were cumulative).

\section{$2.4 \quad$ Covariates}

Covariates were selected based on their relevance to adults with epilepsy, NTFx, and mortality, as well as availability in the administrative claims databases. Sociodemographic variables included age, sex, race, and US region of residence (West, Midwest, South, and Northeast). Baseline comorbidities were identified from 2011 to 2015 by at least (1) one inpatient claim within the 12 months prior to the start date of follow-up; or (2) two outpatient claims on different claim days within 365 days of one another, with the first outpatient claim occurring within the 12 months prior to the start date of follow-up. ${ }^{19}$ Comorbidities included cardiovascular disease (ie, ischemic heart disease, heart failure, cerebrovascular disease), hypertension, diabetes (ie, type 1 , type 2 ), respiratory disease (ie, acute respiratory infection, pneumonia, chronic obstructive pulmonary disease, interstitial/pleura disease, other respiratory disease), chronic kidney disease (stages I-V), and cancer anywhere in the body, as previously described. ${ }^{17,24}$

\section{5 | Statistical analysis}

Descriptive characteristics at baseline were summarized for each group. MRs were estimated as the number of deaths divided by the number of person-years, and expressed per 100 person-years for each group. MR ratios (MRRs) and 95\% confidence intervals (CIs) were estimated comparing the groups to one another.

Cox regression was used to adjust for all covariates when comparing MR, by estimating hazard ratios (HRs) and $95 \%$ CIs of mortality at all time points, comparing the groups to one another. The primary group comparisons of interest were: (1) EP + NTFx versus EP without NTFx to determine whether NTFx is a risk factor for mortality among adults with epilepsy and (2) EP + NTFx versus without EP + NTFx to determine whether NTFx exacerbates MR for adults with versus without epilepsy. Possible interactions between exposure status and age or sex were assessed by conducting separate analyses for age or sex strata (to estimate group effects) and by including product terms in the Cox models (to test for 
interactions). Individuals were right censored if they discontinued health plan enrollment or were alive at the end of the study period.

We then examined crude MR and MRR and adjusted HR of 12-month mortality by NTFx location to identify site-specific effects using the procedures described above.

\section{6 | Sensitivity analysis}

Cox regression did not adjust for race to limit bias due to missing data $(\sim 14 \%)$. We therefore conducted two related sensitivity analyses to assess possible confounding and selection bias. Sensitivity analysis \#1 involved the restricted study population with complete data on race but not adjusting for race; sensitivity analysis \#2 involved the same study population as in \#1 but adjusted for race. Results were compared from sensitivity analyses \#1 and \#2 to assess possible confounding by race. Results were also compared from sensitivity analysis \#1 (restricted study population not adjusting for race) and the main analysis (full study population not adjusting for race) to assess possible selection bias from exclusion of adults without race data.

Analyses were performed using SAS version 9.4 (SAS Institute).

\section{$3 \mid$ RESULTS}

Baseline descriptive characteristics of EP + NTFx ( $\mathrm{n}=11$ 471), EP without NTFx ( $\mathrm{n}=50$ 384), without EP + NTFx ( $=423$ 041), and without EP without NTFx ( $n=6787743$ ) is presented in Table 1. Notably, the two NTFx groups were older and had a higher proportion of women than the two non-NTFx groups.

\subsection{Crude MR}

The crude MRs and MRRs are presented in Table 2. The crude MRs for all time points were highest for EP + NTFx (eg, 12-month $\mathrm{MR}=8.79$ per 100 person-years), followed by without EP + NTFx (eg, 12-month MR = 4.80), EP without NTFx (eg, 12-month MR = 3.06), and without EP without NTFx (eg, 12-month MR =0.47). The MR remained relatively stable for the two non-NTFx groups from 3 months to 12 months, but declined in the two NTFx groups. The crude MRR was elevated for EP + NTFx compared to EP without NTFx and without EP + NTFx for all time points. When without EP without NTFx was the reference, the crude MRR was elevated for all groups for all time points, with a larger decline in MRR for the two NTFx groups compared to the EP without NTFx group.

\section{2 | Cox regression analysis of mortality}

Adjusted mortality HRs are presented in Table 3. Compared to without EP without NTFx, the HR adjusting for all covariates was highest for EP + NTFx across all time points (eg, 12-month HR = 5.23, 95\% CI = 4.88-5.60), followed by without EP + NTFx (eg, 12-month HR = 3.71, 95\% $\mathrm{CI}=3.64-3.78)$ and EP without NTFx (eg, 12-month $\mathrm{HR}=3.08,95 \% \mathrm{CI}=2.92-3.25)$. The adjusted HR comparing EP + NTFx to EP without NTFx was elevated for all time points, with the group effect becoming smaller from 3 months $(\mathrm{HR}=2.87,95 \% \mathrm{CI}=2.47-3.33)$ to 12 months $(\mathrm{HR}=1.70,95 \% \mathrm{CI}=1.58-1.85)$. The adjusted HR comparing EP + NTFx to without EP + NTFx was elevated for all time points, with the group effect becoming larger from 3 months $(\mathrm{HR}=1.11,95 \% \mathrm{CI}=1.00-1.23)$ to 12 months $(\mathrm{HR}=1.41,95 \% \mathrm{CI}=1.32-1.51)$.

\subsection{Site-specific effect of NTFx on 12-month MR}

Table 4 shows the MR, MRR, and adjusted HR for 12month mortality stratified by NTFx location. The sitespecific patterns of MR were consistent for the two NTFx groups; hip had the highest MR, followed by vertebral column and extremities. The crude MR and MRR were elevated for adults with versus without epilepsy for all sites. After adjusting for all covariates, the HR for adults with versus without epilepsy was elevated for all sites, with the lower extremities $(\mathrm{HR}=1.92,95 \% \mathrm{CI}=1.61-2.30)$ and upper extremities $(\mathrm{HR}=1.79,95 \% \mathrm{CI}=1.51-2.12)$ having the highest HR.

\subsection{Interactions of group with age and sex}

Table 5 (age) and Table 6 (sex) show the MR, MRR, and adjusted HR of mortality by age category (18-40, 41-64, and $\geq 65$ years) and sex (both $P$ for interaction $<.001$ ). There were too few deaths in the EP without NTFx and $\mathrm{EP}+$ NTFx groups for the 18-40 year age category for analyses $(\mathrm{n} \leq 40)$. EP + NTFx had an elevated MR, MRR, and adjusted HR compared to EP without NTFx and without EP + NTFx for the 41-64 year and $\geq 65$ year age groups and for both women and men. The $\geq 65$ year age group for all groups by epilepsy and NTFx status had higher MR than the 18-40 and 41-64 year age groups. The absolute difference in MR for EP + NTFx versus EP without NTFx and without EP + NTFx was larger in the $\geq 65$ year versus younger age groups, but the relative MRR was larger for EP + NTFx versus EP without NTFx and without $\mathrm{EP}+\mathrm{NTFx}$ in the 41-64 year versus $\geq 65$ year age group. 


\begin{tabular}{|c|c|c|c|c|}
\hline & $\begin{array}{l}\text { EP + NTFx } \\
n=11471\end{array}$ & $\begin{array}{l}\text { EP without } \\
\text { NTFx, } \\
\text { n=50 } 384\end{array}$ & $\begin{array}{l}\text { Without } \\
\text { EP + NTFx, } \\
n=423041\end{array}$ & $\begin{array}{l}\text { Without EP } \\
\text { without NTFx, } \\
n=6787743\end{array}$ \\
\hline \multicolumn{5}{|c|}{ Demographic characteristics } \\
\hline Age, mean (SD) & $67.1(15.9)$ & $57.6(18.6)$ & $65.0(18.5)$ & $51.7(18.5)$ \\
\hline $18-40$ y, \% & 7.1 & 20.3 & 12.7 & 30.5 \\
\hline $41-64$ y, \% & 31.1 & 38.9 & 28.4 & 41.1 \\
\hline$\geq 65 \mathrm{y}, \%$ & 61.8 & 40.8 & 58.9 & 28.5 \\
\hline \multicolumn{5}{|l|}{ Sex,$\%$} \\
\hline Women & 62.4 & 53.4 & 66.2 & 51.4 \\
\hline Men & 37.6 & 46.6 & 33.8 & 48.6 \\
\hline \multicolumn{5}{|l|}{ Race, $\%$} \\
\hline White & 66.2 & 63.9 & 68.9 & 63.7 \\
\hline Black & 9.4 & 11.1 & 6.5 & 8.4 \\
\hline Hispanic & 7.3 & 8.6 & 7.9 & 9.7 \\
\hline Asian & 1.7 & 2.2 & 2.5 & 4.2 \\
\hline Unknown/missing & 15.4 & 14.2 & 14.2 & 14.0 \\
\hline \multicolumn{5}{|l|}{ US region, $\%$} \\
\hline West & 26.1 & 23.2 & 28.2 & 24.1 \\
\hline Midwest & 22.3 & 23.4 & 24.5 & 25.2 \\
\hline South & 38.9 & 41.1 & 36.2 & 40.5 \\
\hline Northeast & 12.7 & 12.3 & 11.2 & 10.3 \\
\hline \multicolumn{5}{|l|}{ Comorbidities, $\%$} \\
\hline Cardiovascular disease $^{\mathrm{a}}$ & 43.4 & 28.7 & 21.7 & 7.8 \\
\hline Hypertension & 65.4 & 49.0 & 51.7 & 27.5 \\
\hline Diabetes & 25.6 & 20.4 & 19.9 & 11.5 \\
\hline Respiratory disease $^{\mathrm{b}}$ & 41.9 & 30.3 & 27.4 & 14.8 \\
\hline Chronic kidney disease & 15.0 & 9.4 & 10.5 & 3.5 \\
\hline Cancer & 24.1 & 19.2 & 19.2 & 11.2 \\
\hline \multicolumn{5}{|l|}{ Fracture distribution $^{c}$} \\
\hline Unspecified location & 2.1 & & 4.5 & \\
\hline Vertebral column & 30.1 & & 25.4 & \\
\hline Hip & 22.7 & & 16.7 & \\
\hline Femur, nonproximal & 3.6 & & 3.1 & \\
\hline Tibia/fibula & 18.0 & & 22.9 & \\
\hline Humerus & 12.5 & & 9.8 & \\
\hline Ulna/radius & 12.8 & & 19.2 & \\
\hline
\end{tabular}

TA B L E 1 Baseline descriptive characteristics by status of EP and NTFx

Abbreviations: EP, epilepsy; NTFx, nontrauma fracture.

${ }^{\mathrm{a}}$ Ischemic heart disease, heart failure, and/or cerebrovascular disease.

${ }^{\mathrm{b}}$ Acute respiratory infection, pneumonia, chronic obstructive pulmonary disease, interstitial/pleura disease,

and/or other respiratory disease (eg, respiratory failure).

${ }^{\mathrm{c}}$ Some individuals had an NTFx across multiple sites.

Men had a higher MR for all groups compared to women. The absolute difference in MR was larger for men than women for EP + NTFx versus EP without NTFx and without EP + NTFx, and the relative MRR was larger for men than women for EP + NTFx versus EP without NTFx.

\section{5 | Sensitivity analysis}

There were 6257223 individuals with complete data on race. The adjusted HR was higher for EP + NTFx versus EP without NTFx for 3 months (covariate adjusted and 


\begin{tabular}{|c|c|c|c|c|}
\hline Mortality cases & \multicolumn{2}{|c|}{ 3-mo mortality, n } & 6-mo mortality, n & $\begin{array}{l}\text { 12-mo } \\
\text { mortality, n }\end{array}$ \\
\hline Without EP without NTFx & \multicolumn{2}{|l|}{7150} & 14297 & 28505 \\
\hline Without EP + NTFx & \multicolumn{2}{|l|}{9003} & 12656 & 17397 \\
\hline EP without NTFx & \multicolumn{2}{|l|}{324} & 668 & 1399 \\
\hline $\mathrm{EP}+\mathrm{NTFx}$ & \multicolumn{2}{|l|}{354} & 579 & 856 \\
\hline Crude mortality rate & \multicolumn{2}{|l|}{$\begin{array}{l}\text { Per } 100 \\
\text { person-years, } n\end{array}$} & $\begin{array}{l}\text { Per } 100 \\
\text { person-years, } n\end{array}$ & $\begin{array}{l}\text { Per } 100 \\
\text { person-years, } n\end{array}$ \\
\hline Without EP without NTFx & \multicolumn{2}{|l|}{0.43} & 0.45 & 0.47 \\
\hline Without EP + NTFx & \multicolumn{2}{|l|}{8.90} & 6.51 & 4.80 \\
\hline EP without NTFx & \multicolumn{2}{|l|}{2.63} & 2.77 & 3.06 \\
\hline $\mathrm{EP}+\mathrm{NTFx}$ & \multicolumn{2}{|l|}{12.89} & 11.01 & 8.79 \\
\hline Crude mortality RR & $\mathbf{R R}(\mathbf{9 5 \%} \mathbf{C I})$ & \multicolumn{2}{|c|}{$\mathbf{R R}(\mathbf{9 5} \% \mathbf{C I})$} & RR $(95 \%$ CI $)$ \\
\hline \multicolumn{5}{|c|}{ Reference: without EP without NTFx } \\
\hline Without EP + NTFx & $20.54(19.91-21.19)$ & \multicolumn{2}{|c|}{$14.62(14.27-14.97)$} & $10.23(10.03-10.41)$ \\
\hline EP without NTFx & $6.07(5.43-6.78)$ & 6.2 & $(5.76-6.73)$ & $6.51(6.17-6.87)$ \\
\hline $\mathrm{EP}+\mathrm{NTFx}$ & $29.75(26.74-33.10)$ & 24. & $1(22.74-26.85)$ & $18.72(17.49-20.03)$ \\
\hline Reference: without EP + N & NTFx & & & \\
\hline EP without NTFx & $0.30(0.26-0.33)$ & 0.4 & $(0.39-0.46)$ & $0.64(0.60-0.67)$ \\
\hline $\mathrm{EP}+\mathrm{NTFx}$ & $1.45(1.30-1.61)$ & 1.6 & $(1.56-1.84)$ & $1.83(1.71-1.96)$ \\
\hline Reference: EP without NT & $\Gamma F x$ & & & \\
\hline $\mathrm{EP}+\mathrm{NTFx}$ & $4.91(4.22-5.70)$ & 3.9 & $(3.55-4.44)$ & $2.88(2.64-3.13)$ \\
\hline
\end{tabular}

Abbreviations: CI, confidence interval; EP, epilepsy; NTFx, nontrauma fracture; RR, rate ratio.

\begin{tabular}{|llll} 
& $\begin{array}{l}\text { 3-mo mortality, } \\
\text { HR (95\% CI) }\end{array}$ & $\begin{array}{l}\text { 6-mo mortality, } \\
\text { HR (95\% CI) }\end{array}$ & $\begin{array}{l}\text { 12-mo mortality, } \\
\text { HR (95\% CI) }\end{array}$ \\
\hline $\begin{array}{l}\text { Reference: without EP without NTFx } \\
\text { Without EP + NTFx }\end{array}$ & $7.19(6.96-7.43)$ & $5.20(5.07-5.33)$ & $3.71(3.64-3.78)$ \\
EP without NTFx & $2.78(2.48-3.11)$ & $2.88(2.66-3.11)$ & $3.08(2.92-3.25)$ \\
EP + NTFx & $7.96(7.15-8.87)$ & $6.73(6.19-7.32)$ & $5.23(4.88-5.60)$ \\
\hline Reference: without EP + NTFx & & & \\
\hline EP without NTFx & $0.39(0.35-0.43)$ & $0.55(0.51-0.60)$ & $0.83(0.79-0.88)$ \\
EP + NTFx & $1.11(1.00-1.23)$ & $1.29(1.19-1.41)$ & $1.41(1.32-1.51)$ \\
\hline Reference: EP without NTFx & & & \\
EP + NTFx & $2.87(2.47-3.33)$ & $2.34(2.09-2.61)$ & $1.70(1.58-1.85)$ \\
\hline
\end{tabular}

Note: Adjusted for age, sex, US region, cardiovascular disease, respiratory disease, diabetes, chronic kidney disease, cancer, and hypertension.

Abbreviations: CI, confidence interval; EP, epilepsy; HR, hazard ratio; NTFx, nontrauma fracture.
TA B LE 2 Crude mortality rate and RR by status of EP and NTFx
T A B L E 3 Adjusted HR of mortality by status of EP and NTFx covariate + race HR rounded to the same values; both $\mathrm{HR}=2.98,95 \% \mathrm{CI}=2.54-3.50$ ), 6 months (covariate adjusted $\mathrm{HR}=2.33,95 \% \mathrm{CI}=2.07-2.62$; covariate + race $\mathrm{HR}=2.34,95 \% \mathrm{CI}=2.08-2.63$ ), and 12 months (covariate adjusted $\mathrm{HR}=1.71,95 \% \mathrm{CI}=1.56-1.88$; covariate + race $\mathrm{HR}=1.72,95 \% \mathrm{CI}=1.57-1.88)$. The adjusted $\mathrm{HR}$ was higher for EP + NTFx versus without EP + NTFx for 3 months (covariate adjusted HR $=1.13,95 \%$ CI $=1.01-1.27$; covariate + race $\mathrm{HR}=1.12,95 \% \mathrm{CI}=1.00-1.25), 6$ months (covariate adjusted $\mathrm{HR}=1.31,95 \% \mathrm{CI}=1.20-1.43$; covariate + race $\mathrm{HR}=1.29,95 \% \mathrm{CI}=1.18-1.41)$, and 12 months (covariate adjusted $\mathrm{HR}=1.43,95 \% \mathrm{CI}=1.33-1.54$; covariate + race $\mathrm{HR}=1.41,95 \% \mathrm{CI}=1.31-1.52)$. A comparison of the HR estimates from sensitivity analyses \#1 and \#2 yielded similar results, suggesting that race is not a confounder in the main analysis. A comparison of HR 
TA B L E 4 Twelve-month mortality rate, $\mathrm{RR}$, and adjusted $\mathrm{HR}$ by status of $\mathrm{EP}$ and NTFx location

\begin{tabular}{|lllll|}
\hline Mortality cases & $\begin{array}{l}\text { Vertebral } \\
\text { column, } \mathbf{n}\end{array}$ & Hip, $\mathbf{n}$ & $\begin{array}{l}\text { Lower } \\
\text { extremities, } \mathbf{n}\end{array}$ & $\begin{array}{l}\text { Upper } \\
\text { extremities, } \mathbf{n}\end{array}$ \\
\hline Without EP + NTFx & 6391 & 6420 & 1951 & 2514 \\
\hline EP + NTFx & 289 & 281 & 129 & 145 \\
& & Per 100 & & \\
& Per 100 person- & person-years, & Per 100 person- & Per 100 person- \\
Crude mortality rate & years, $\mathbf{n}$ & $\mathbf{n}$ & years, $\mathbf{n}$ & years, $\mathbf{n}$ \\
\hline Without EP + NTFx & 7.01 & 11.22 & 2.06 & 2.35 \\
\hline EP + NTFx & 9.93 & 13.34 & 6.00 & 5.76 \\
\hline Crude mortality RR & RR (95\% CI) & RR (95\% CI) & RR (95\% CI) & RR (95\% CI) \\
\hline
\end{tabular}

Reference: without EP + NTFx

\begin{tabular}{ccccc} 
EP + NTFx & $1.42(1.26-1.59)$ & $1.19(1.06-1.34)$ & $2.92(2.44-3.49)$ & $2.45(2.07-2.90)$ \\
\hline Adjusted HR & HR (95\% CI) & HR (95\% CI) & HR (95\% CI) & HR (95\% CI) \\
\hline $\begin{array}{l}\text { Reference: without EP + NTFx } \\
\text { EP + NTFx }\end{array}$ & $1.30(1.16-1.47)$ & $1.15(1.02-1.30)$ & $1.92(1.61-2.30)$ & $1.79(1.51-2.12)$ \\
\hline
\end{tabular}

Note: Adjusted for age, sex, US region, cardiovascular disease, respiratory disease, diabetes, chronic kidney disease, cancer, and hypertension.

Abbreviations: CI, confidence interval; EP, epilepsy; HR, hazard ratio; NTFx, nontrauma fracture; RR, rate ratio.

estimates from sensitivity analysis \#1 with results from the main analysis show similar results, suggesting no evidence of selection bias.

\section{4 | DISCUSSION}

Findings from this study suggest that the elevated mortality burden for adults with epilepsy ${ }^{15}$ may be exacerbated by skeletal fragility. One main finding of this investigation is that NTFx was associated with mortality within 12 months among adults with epilepsy, suggesting that NTFx is a risk factor for mortality for this population. The other main finding of this investigation was that among those who sustained an NTFx, adults with epilepsy had higher rates of mortality within 12 months compared to adults without epilepsy, suggesting a greater NTFx-attributable mortality burden for adults with epilepsy. These findings were evident across NTFx sites and after accounting for covariates that are associated with premature mortality. Taken together, study findings suggest that NTFx, an indicator of skeletal fragility, may be implicated in the pathogenesis of unhealthful aging for adults with epilepsy.

A recent systematic review including 46 studies concluded that individuals with epilepsy are at risk for premature mortality. ${ }^{15}$ Although the burden of premature mortality varied among epilepsy-related factors (eg, etiology), the standardized mortality ratios were still elevated for most studies regardless of age, sex, etiology, type, and time since diagnosis. In the elderly general population, fracture is associated with mortality ${ }^{7,10,11}$ and chronic diseases, ${ }^{8,25}$ and postfracture chronic disease development may be involved in the fracture-mortality association. ${ }^{8,25,26}$ The most common causes of death for individuals with epilepsy include chronic diseases (eg, cardiovascular and respiratory disease), injuries (eg, seizures, falls), and cancer. ${ }^{15,27-29}$ Therefore, the current study provides new potential insights into factors that may be involved directly with mortality or indirectly with mortality via early pathological processes for adults with epilepsy, as NTFx is an adverse outcome of injuries (eg, seizures, falls) and increases risk of chronic diseases.

In the current study, the crude analysis found that MR was highest among EP + NTFx, followed by without EP + NTFx, EP without NTFx, and without EP without NTFx. Interestingly, the non-NTFx groups had a relatively consistent MR across the 3-, 6-, and 12-month time points, whereas the two NTFx groups had the highest MR at 3 months and a gradual decline to 12 months. Even with this decline, the MR was higher for all time points compared to the non-NTFx groups. This finding is consistent with the conviction that there may be a unique set of factors associated with shortand long-term mortality post-NTFx. ${ }^{30}$ However, the mechanisms explaining the NTFx-induced mortality have yet to be elucidated and may be further confounded by the medical complexities of epilepsy (eg, antiseizure medications).

We used Cox regression to adjust for demographics and several chronic diseases that are associated with NTFx and mortality to better understand the link between epilepsy, NTFx, and mortality beyond these covariates. In our primary comparisons of interest, we found that the adjusted rate of 3-, 6-, and 12-month mortality was higher for EP + NTFx compared to EP without 


\begin{tabular}{|c|c|c|c|}
\hline Mortality cases & $18-40 y, n$ & $41-64 y, n$ & $\geq 65 y, n$ \\
\hline without EP without NTFx & 312 & 3612 & 24581 \\
\hline without EP + NTFx & 64 & 1325 & 16008 \\
\hline EP without NTFx & 40 & 292 & 1067 \\
\hline $\mathrm{EP}+\mathrm{NTFx}$ & $\leq 10$ & 149 & 699 \\
\hline Crude mortality rate & $\begin{array}{l}\text { Per } 100 \text { person- } \\
\text { years, } n\end{array}$ & $\begin{array}{l}\text { Per } 100 \text { person- } \\
\text { years, } n\end{array}$ & $\begin{array}{l}\text { Per } 100 \text { person- } \\
\text { years, } n\end{array}$ \\
\hline without EP without NTFx & 0.02 & 0.15 & 1.38 \\
\hline without EP + NTFx & 0.14 & 1.28 & 7.50 \\
\hline EP without NTFx & 0.44 & 1.64 & 5.70 \\
\hline $\mathrm{EP}+\mathrm{NTFx}$ & 1.14 & 4.81 & 11.80 \\
\hline Crude mortality RR & $\mathbf{R R}(\mathbf{9 5} \% \mathbf{C I})$ & $\mathbf{R R}(\mathbf{9 5} \% \mathbf{C I})$ & $\mathbf{R R}(\mathbf{9 5 \%} \mathbf{C I})$ \\
\hline \multicolumn{4}{|c|}{ Reference: without EP without NTFx } \\
\hline Without EP + NTFx & $8.09(6.18-10.58)$ & $8.81(8.27-9.38)$ & $5.46(5.35-5.57)$ \\
\hline EP without NTFx & a & $11.24(9.98-12.66)$ & $4.15(3.90-4.41)$ \\
\hline $\mathrm{EP}+\mathrm{NTFx}$ & a & $33.04(28.05-38.92)$ & $8.58(7.96-9.25)$ \\
\hline \multicolumn{4}{|c|}{ Reference: without EP + NTFx } \\
\hline EP without NTFx & a & $1.28(1.12-1.45)$ & $0.76(0.72-0.81)$ \\
\hline $\mathrm{EP}+\mathrm{NTFx}$ & a & $3.75(3.17-4.45)$ & $1.57(1.46-1.70)$ \\
\hline \multicolumn{4}{|l|}{ Reference: EP without NTFx } \\
\hline $\mathrm{EP}+\mathrm{NTFx}$ & a & $2.94(2.41-3.58)$ & $2.07(1.88-2.28)$ \\
\hline Adjusted HR & HR $(95 \%$ CI $)$ & HR $(95 \%$ CI $)$ & HR $(95 \%$ CI $)$ \\
\hline \multicolumn{4}{|c|}{ Reference: without EP without NTFx } \\
\hline Without EP + NTFx & $6.32(4.81-8.31)$ & $5.21(4.88-5.57)$ & $3.48(3.41-3.55)$ \\
\hline EP without NTFx & a & $4.43(3.92-5.01)$ & $2.65(2.49-2.82)$ \\
\hline $\mathrm{EP}+\mathrm{NTFx}$ & a & $8.78(7.43-10.38)$ & $4.54(4.21-4.90)$ \\
\hline \multicolumn{4}{|c|}{ Reference: without EP + NTFx } \\
\hline EP without NTFx & a & $0.85(0.75-0.97)$ & $0.76(0.72-0.81)$ \\
\hline $\mathrm{EP}+\mathrm{NTFx}$ & a & $1.68(1.42-2.00)$ & $1.30(1.21-1.41)$ \\
\hline \multicolumn{4}{|l|}{ Reference: EP without NTFx } \\
\hline $\mathrm{EP}+\mathrm{NTFx}$ & a & $1.98(1.63-2.41)$ & $1.71(1.56-1.88)$ \\
\hline
\end{tabular}

TA B LE 5 Twelve-month mortality rate, RR, and adjusted HR by status of EP and NTFx by age categories

Note: Adjusted for age, sex, US region, cardiovascular disease, respiratory disease, diabetes, chronic kidney disease, cancer, and hypertension.

Abbreviations: CI, confidence interval; EP, epilepsy; HR, hazard ratio; NTFx, nontrauma fracture; RR, rate ratio.

${ }^{\mathrm{a}}$ Sample size was insufficient for analysis.

NTFx and without EP + NTFx. Specifically, EP + NTFx had a 2.9-fold higher rate of mortality in the 3 months following the NTFx event compared to EP without NTFx. The adjusted risk declined over the time points, but still remained elevated, with a 70\% higher adjusted rate of mortality at 12 months. The adjusted rate of mortality for EP + NTFx compared to without $\mathrm{EP}+\mathrm{NTFx}$ increased over the time points, from $11 \%$ higher rate at 3 months to $41 \%$ higher rate at 12 months.

When we stratified NTFx by location, we found that all sites were associated with higher adjusted 12-month MR among adults with versus without epilepsy. However, we found that
NTFx of the lower and upper extremities was associated with higher relative MRs compared to the hip and vertebral column (92\%, 79\%, 15\%, and 30\%, respectively). However, it is important to note that the absolute MR was still much higher for NTFx of the hip and vertebral column than extremities for adults with and without epilepsy. Therefore, NTFx at all of these sites should be treated with urgency (eg, aggressive monitoring and treatment interventions) for adults with epilepsy, and especially if NTFx occurs in the lower or upper extremities.

Finally, we found that the excess mortality burden attributable to NTFx for adults with epilepsy from the whole 
TA B LE 6 Twelve-month mortality rate, $\mathrm{RR}$, and adjusted $\mathrm{HR}$ by status of $\mathrm{EP}$ and NTFx by sex

\begin{tabular}{|c|c|c|}
\hline Mortality cases & Women, $\mathbf{n}$ & Men, $\mathbf{n}$ \\
\hline Without EP without NTFx & 13773 & 14732 \\
\hline Without EP + NTFx & 10343 & 7054 \\
\hline EP without NTFx & 682 & 717 \\
\hline $\mathrm{EP}+\mathrm{NTFx}$ & 464 & 392 \\
\hline Crude mortality rate & Per 100 person-years, $n$ & Per 100 person-years, $n$ \\
\hline Without EP without NTFx & 0.44 & 0.50 \\
\hline Without EP + NTFx & 4.27 & 5.87 \\
\hline EP without NTFx & 2.80 & 3.36 \\
\hline $\mathrm{EP}+\mathrm{NTFx}$ & 7.52 & 10.99 \\
\hline \multicolumn{2}{|c|}{ Crude mortality RR } & $\mathbf{R R}(\mathbf{9 5} \% \mathbf{C I})$ \\
\hline \multicolumn{3}{|c|}{ Reference: without EP without NTFx } \\
\hline Without EP + NTFx & $9.66(9.42-9.91)$ & $11.76(11.43-12.10)$ \\
\hline EP without NTFx & $6.33(5.86-6.84)$ & $6.72(6.23-7.24)$ \\
\hline $\mathrm{EP}+\mathrm{NTFx}$ & $17.03(15.53-18.68)$ & $22.00(19.90-24.32)$ \\
\hline \multicolumn{3}{|c|}{ Reference: without EP + NTFx } \\
\hline EP without NTFx & $0.66(0.61-0.71)$ & $0.57(0.53-0.62)$ \\
\hline $\mathrm{EP}+\mathrm{NTFx}$ & $1.76(1.61-1.94)$ & $1.87(1.69-2.07)$ \\
\hline \multicolumn{3}{|l|}{ Reference: EP without NTFx } \\
\hline $\mathrm{EP}+\mathrm{NTFx}$ & $2.69(2.39-3.03)$ & $3.28(2.90-3.70)$ \\
\hline Adjusted HR & HR $(95 \%$ CI $)$ & HR $(95 \%$ CI $)$ \\
\hline \multicolumn{3}{|c|}{ Reference: without EP without NTFx } \\
\hline Without EP + NTFx & $3.20(3.12-3.29)$ & $4.43(4.30-4.57)$ \\
\hline EP without NTFx & $3.05(2.82-3.29)$ & $3.11(2.88-3.35)$ \\
\hline $\mathrm{EP}+\mathrm{NTFx}$ & $4.35(3.97-4.78)$ & $6.39(5.78-7.07)$ \\
\hline \multicolumn{3}{|c|}{ Reference: without EP + NTFx } \\
\hline EP without NTFx & $0.95(0.88-1.03)$ & $0.70(0.65-0.76)$ \\
\hline $\mathrm{EP}+\mathrm{NTFx}$ & $1.36(1.24-1.49)$ & $1.44(1.30-1.60)$ \\
\hline Reference: EP without NTF & & \\
\hline $\mathrm{EP}+\mathrm{NTFx}$ & $1.43(1.27-1.61)$ & $2.06(1.82-2.33)$ \\
\hline
\end{tabular}

Note: Adjusted for age, US region, cardiovascular disease, respiratory disease, diabetes, chronic kidney disease, cancer, and hypertension.

Abbreviations: CI, confidence interval; EP, epilepsy; HR, hazard ratio; NTFx, nontrauma fracture; RR, rate ratio. sample was consistent across the age spectrum (ie, young, middle-aged, and older) and for women and men. However, we found that the group effect estimate was larger for middle-age than older age for EP + NTFx versus EP without NTFx and without EP + NTFx, and for men than women for EP + NTFx versus EP without NTFx. Taken together, these findings suggest that the NTFX-attributable mortality burden is more problematic for individuals with versus without epilepsy across the adult lifespan and for both women and men, but that younger age subjects and men with epilepsy may have a higher relative vulnerability to the NTFX-mortality burden.

Prefracture diseases, ${ }^{31}$ functional capacity, ${ }^{32}$ and physical ability (eg, muscle strength) ${ }^{33}$ are risk factors for postfracture premature mortality, and a follow-up fracture further exacerbates mortality risk. ${ }^{33}$ The systemic stress induced by an NTFx may create an unfavorable physiological environment that exacerbates risk for post-NTFx complications and premature mortality, through either direct or indirect mechanisms. ${ }^{30}$ Individuals with epilepsy are particularly susceptible to postNTFx complications because of their greater lifetime burden of unhealthful aging, as evidenced by an elevated prevalence of chronic diseases in adulthood. ${ }^{12-14}$ Therefore, adults with epilepsy may already have a compromised physiological environment and skeletal fragility (eg, history of NTFx), and sustaining an NTFx may further exacerbate risk of postNTFx complications and premature mortality. Future studies are needed to disentangle the complex factors involved in 
post-NTFx mortality unique to individuals with epilepsy, and to determine to what extent the NTFx-mortality association is mediated by preventable (eg, serious falls, postoperative complications), modifiable (eg, physical activity), or treatable (eg, chronic disease) factors.

The limitations of this study must be discussed. First, there is a risk of biasing effect estimates from unmeasured confounding due to the limited set of comorbidities, the observational design, and the lack of medication information, as we were not able to ascertain pharmacy claims. In light of these factors, we computed $E$ values ${ }^{34,35}$ to determine the extent of unmeasured confounding (minimum strength of association with the exposure and outcome) needed to fully explain away a specific exposure-outcome association, conditional on the set of covariates. We used the fully adjusted Cox regression model for each analysis. The $E$ value (lower 95\% CI) needed to fully explain away the effect for EP + NTFx versus EP without NTFx was 5.19 (4.38) for 3 months, 4.11 (3.60) for 6 months, and 2.79 (2.54) for 12 months. The $E$ value (lower $95 \%$ CI) needed to fully explain away the effect for EP + NTFx versus without EP + NTFX was 1.46 (1.00) for 3 months, 1.90 (1.67) for 6 months, and 2.17 (1.97) for 12 months. Given the careful selection of covariates in the design of the Cox regression models as well as the large $E$ values, it appears unlikely that unmeasured confounding (eg, lack of medication information) largely biased effect estimates for the exposure variables. Second, we were unable to account for the type, etiology, or time since diagnosis of epilepsy, which may have provided deeper insight into the NTFx-mortality burden. Third, it is not possible to reliably determine the cause of fracture using claims data. Approximately one-third of fractures can be attributable to seizures for adults with epilepsy. ${ }^{36}$ Seizures by themselves may not be considered traumatic and would therefore not be given a trauma code in the claims database (eg, not including seizures that lead to a motor vehicle accident). A fracture not having a trauma code around the date of fracture would be considered an NTFx in the current study. Fourth, epilepsy was not determined using diagnosis codes for a single seizure (ICD-10, G41 family codes) or convulsions (ICD-9, 780.3x; ICD-10, R56.8), in part due to a potential for misclassification of epilepsy; however, misclassification for this reason would have a negligible impact on study findings given the large sample size.

In conclusion, study findings suggest that NTFx is a risk factor for mortality among adults with epilepsy, and compared to adults without epilepsy, NTFx elicits a higher MR for up to 12 months for adults with epilepsy. Furthermore, while NTFx across all sites is associated with an elevated 12month MR for adults with versus without epilepsy, there may be a stronger mortality association with NTFx of the lower and upper extremities. Future clinical research is needed to identify strategies to prevent and better manage skeletal fragility with the aim of reducing the burden of skeletal fragility and improving healthful aging for adults with epilepsy. Future basic and translational studies are needed that examine mechanisms linking skeletal fragility with mortality specific to the population of adults with epilepsy.

\section{ACKNOWLEDGMENTS}

This work was supported by the University of Michigan Office of Health Equity and Inclusion Diversity Fund and the American Academy of Cerebral Palsy and Developmental Medicine. Study sponsors had no role in the design or conduct of the research, or dissemination of the work.

\section{CONFLICT OF INTEREST}

None of the authors has any conflict of interest to disclose. We confirm that we have read the Journal's position on issues involved in ethical publication and affirm that this report is consistent with those guidelines.

\section{ORCID}

Daniel G. Whitney (iD https://orcid. org/0000-0003-2132-1527

\section{REFERENCES}

1. McNamara NA, Romanowski EMF, Olson DP, Shellhaas RA. Bone health and endocrine comorbidities in pediatric epilepsy. Semin Pediatr Neurol. 2017;24(4):301-9.

2. Gniatkowska-Nowakowska A. Fractures in epilepsy children. Seizure. 2010;19(6):324-5.

3. Coppola G, Fortunato D, Auricchio G, et al. Bone mineral density in children, adolescents, and young adults with epilepsy. Epilepsia. 2009;50(9):2140-6.

4. Beerhorst K, Tan IY, De Krom M, Verschuure P, Aldenkamp AP. Antiepileptic drugs and high prevalence of low bone mineral density in a group of inpatients with chronic epilepsy. Acta Neurol Scand. 2013;128(4):273-80.

5. Fedorenko M, Wagner ML, Wu BY. Survey of risk factors for osteoporosis and osteoprotective behaviors among patients with epilepsy. Epilepsy Behav. 2015;45:217-22.

6. Vestergaard P. Epilepsy, osteoporosis and fracture risk-a meta-analysis. Acta Neurol Scand. 2005;112(5):277-86.

7. Neuman MD, Silber JH, Magaziner JS, Passarella MA, Mehta S, Werner RM. Survival and functional outcomes after hip fracture among nursing home residents. JAMA Intern Med. 2014;174(8):1273-80.

8. Veronese N, Stubbs B, Crepaldi G, et al. Relationship between low bone mineral density and fractures with incident cardiovascular disease: a systematic review and meta-analysis. J Bone Miner Res. 2017;32(5):1126-35.

9. Harvey-Kelly KF, Kanakaris NK, Obakponovwe O, West RM, Giannoudis PV. Quality of life and sexual function after traumatic pelvic fracture. J Orthop Trauma. 2014;28(1):28-35.

10. Kumar A, Rahman M, Trivedi AN, Resnik L, Gozalo P, Mor V. Comparing post-acute rehabilitation use, length of stay, and outcomes experienced by Medicare fee-for-service and Medicare Advantage beneficiaries with hip fracture in the United 
States: a secondary analysis of administrative data. PLoS Med. 2018;15(6):e1002592.

11. Uriz-Otano F, Pla-Vidal J, Tiberio-Lopez G, Malafarina V. Factors associated to institutionalization and mortality over three years, in elderly people with a hip fracture - an observational study. Maturitas. 2016;89:9-15.

12. Weatherburn CJ, Heath CA, Mercer SW, Guthrie B. Physical and mental health comorbidities of epilepsy: population-based cross-sectional analysis of 1.5 million people in Scotland. Seizure. 2017;45:125-31.

13. Pham T, Sauro KM, Patten SB, et al. The prevalence of anxiety and associated factors in persons with epilepsy. Epilepsia. 2017;58(8):e107-10.

14. Scott AJ, Sharpe L, Hunt C, Gandy M. Anxiety and depressive disorders in people with epilepsy: a meta-analysis. Epilepsia. 2017;58(6):973-82.

15. Thurman DJ, Logroscino G, Beghi E, et al. The burden of premature mortality of epilepsy in high-income countries: a systematic review from the Mortality Task Force of the International League Against Epilepsy. Epilepsia. 2017;58(1):17-26.

16. Whitney DG, Alford AI, Devlin MJ, Caird MS, Hurvitz EA, Peterson MD. Adults with cerebral palsy have higher prevalence of fracture compared to adults without cerebral palsy independent of osteoporosis and cardiometabolic diseases. J Bone Miner Res. 2019;34:1240-7.

17. Whitney DG, Whibley D, Jepsen KJ. The effect of low-trauma fracture on one-year mortality rate among privately insured adults with and without neurodevelopmental disabilities. Bone. 2019;129:115060.

18. Moura L, Smith JR, Blacker D, Vogeli C, Schwamm LH, Hsu J. Medicare claims can identify post-stroke epilepsy. Epilepsy Res. 2019;151:40-7.

19. Chang HY, Weiner JP, Richards TM, Bleich SN, Segal JB. Validating the adapted Diabetes Complications Severity Index in claims data. Am J Manag Care. 2012;18(11):721-6.

20. Holden EW, Grossman E, Nguyen HT, et al. Developing a computer algorithm to identify epilepsy cases in managed care organizations. Dis Manag. 2005;8(1):1-14.

21. Keshishian A, Boytsov N, Burge R, et al. Examining the effect of medication adherence on risk of subsequent fracture among women with a fragility fracture in the U.S. Medicare Population. J Manag Care Spec Pharm. 2017;23(11):1178-90.

22. Büchele G, Becker C, Cameron ID, et al. Fracture risk in people with developmental disabilities: results of a large claims data analysis. Osteoporos Int. 2017;28(1):369-75.

23. Narongroeknawin P, Patkar NM, Shakoory B, et al. Validation of diagnostic codes for subtrochanteric, diaphyseal, and atypical femoral fractures using administrative claims data. J Clin Densitom. 2012;15(1):92-102.

24. Whitney DG. Prevalence of high-burden medical conditions among young and middle-aged adults with pediatric-onset medical conditions: findings from US private and public administrative claims data. Int J Health Policy Manag. 2019;8(11):629-35.

25. Inoue D, Watanabe R, Okazaki R. COPD and osteoporosis: links, risks, and treatment challenges. Int J Chron Obstruct Pulmon Dis. 2016;11:637-48.

26. Coutinho ES, Bloch KV, Coeli CM. One-year mortality among elderly people after hospitalization due to fall-related fractures: comparison with a control group of matched elderly. Cad Saude Publica. 2012;28(4):801-5.

27. Chamorro-Munoz MI, Garcia-Martin G, Perez-Errazquin F, Romero-Acebal M, Garcia-Rodriguez A, Gutierrez-Bedmar M. Epidemiological study of mortality in epilepsy in a Spanish population. Seizure. 2017;46:19-23.

28. Keezer MR, Bell GS, Neligan A, Novy J, Sander JW. Cause of death and predictors of mortality in a community-based cohort of people with epilepsy. Neurology. 2016;86(8):704-12.

29. Chang C-Y, Lu T-H, Cheng T-J. Trends in reporting injury as a cause of death among people with epilepsy in the U.S., 1981-2010. Seizure. 2014;23(10):836-43.

30. Schousboe JT. Mortality after osteoporotic fractures: what proportion is caused by fracture and is preventable? J Bone Miner Res. 2017;32(9):1783-8.

31. Katsoulis M, Benetou V, Karapetyan T, et al. Excess mortality after hip fracture in elderly persons from Europe and the USA: the CHANCES project. J Intern Med. 2017;281(3):300-10.

32. Tosteson AN, Gottlieb DJ, Radley DC, Fisher ES, Melton LJ III. Excess mortality following hip fracture: the role of underlying health status. Osteoporos Int. 2007;18(11):1463-72.

33. Bliuc D, Nguyen ND, Milch VE, Nguyen TV, Eisman JA, Center JR. Mortality risk associated with low-trauma osteoporotic fracture and subsequent fracture in men and women. JAMA. 2009;301(5):513-21.

34. Mathur MB, Ding P, Riddell CA, VanderWeele TJ. Website and R package for computing E-values. Epidemiology. 2018;29(5):e45-7.

35. VanderWeele TJ, Ding P. Sensitivity analysis in observational research: introducing the E-value. Ann Intern Med. 2017;167(4):268-74.

36. Vestergaard P, Tigaran S, Rejnmark L, Tigaran C, Dam M, Mosekilde L. Fracture risk is increased in epilepsy. Acta Neurol Scand. 1999;99(5):269-75.

How to cite this article: Whitney DG, Bell S, McNamara NA, Hurvitz EA. The mortality burden attributable to nontrauma fracture for privately insured adults with epilepsy. Epilepsia. 2020;61:714-724. https://doi.org/10.1111/epi.16465 